$\xi=-1$

\title{
Coalescention of Water Oil Emulsions on the Surface of Ptfe Grains
}

\author{
Dinar D. Fazullin, Gennady V. Mavrin, Ilnar A. Nasyrov, Mikhail P. Sokolov \\ Kazan Federal University \\ *Corresponding author E-mail:denr3@yandex.ru
}

\begin{abstract}
In this work researches on division of water oil emulsions and oil-containing waste by a coalescention method on granular polymers from a polytetrafluoroethylene (PTFE) with sizes of particles of grains from $0,5 \mathrm{~mm}$ to $3 \mathrm{~mm}$ are conducted. Dynamic filtration of an emulsion was carried out through grains of polymer filled in a column with a diameter of $10 \mathrm{~mm}$, the mass of grains of polymer was $2 \mathrm{~g}$, and height of filling of a column of 10-12 cm. Speed of filtration of these emulsions through a column depends on the size of grains of a polytetrafluoroethylene. So with increase in the size of particles the speed of filtration increases, but the efficiency of remo val of oil products decreases. Extent of cleaning of a water oil emulsion of oil products with initial concentration of $828 \mathrm{mg} / \mathrm{dm} 3 \mathrm{made} 62 \%$ at filtration through polymer with sizes of grains $<0,5 \mathrm{~mm}$ and $51 \%$ at filtration of an emulsion through polymer grains with a size more than $3 \mathrm{~mm}$. The efficiency of removal of free oil products was more than $90 \%$ of waste water. On the surface of grains of polymers the coalescention and aggregation of a particle of oil products is observed. Coalescention of oil products on grains of polymer is effective at removal from the unstable and stratified emulsions.
\end{abstract}

Keywords Water oil emulsion, oil products, water-repellence, coalescention, polytetrafluoroethylene, efficiency of cleaning, filtration speed

\section{Introduction}

In sewage oil products to be in the free, emulsified and dissolved states. Free oil products and large particles of oil products, oils, are removed as a result of upholding in settlers and petrotraps. For removal of the emulsified oil products, with sizes of particles of oil products from 0,1 to 10 microns traditionally use floatation ways of cleaning, methods of electrothermic coagulation and electroflotation. But these methods of cleaning demand power expenses and have the difficult technical device. Also these methods of cleaning do not allow deleting oil products completely. Deep cleaning of particles of the oil products with sizes less than $1 \mathrm{mi}-$ cron which are especially emulsified oil products to concentration less than $10 \mathrm{mg} / \mathrm{dm} 3$ it is reached in processes of filtration and membrane division generally by means of microfiltration and ultrafiltration [1-3].

Coalescention is simple, inexpensive and safe process of division of water oil emulsions [4-8]. Process of a coalescention proceeds independently, does not demand additional power expenses, except supply of liquid in the coalescention camera. Devices for a coalescention are arranged simply, have no moving and rotating parts, and in the course of cleaning chemical reagents are not used. Nozzles for a coalescention can be arranged in the form of thin layer plates, hollow tubes, fibers, granules, grains, flakes [8-11].

For example, in works $[10,12]$ installations of separation of an oil phase from an emulsion like oil in water are developed. In these devices process of a coalescention happens between thin layer plates. As material of thin layer plates polyvinylchloride, polypro- pylene, PTFE, nylon-66 is used. Space between two plates quite narrow to improve collisions between oil drops and with a plate surface. Drops of oil have affinity to a surface of a plate and, thus, are taken, and then stick together on a surface, after integration of particles of oil on the surface of polymer there is an education and office of drops of oil which drops on a surface and further from a surface are removed mechanically or are poured in capacity for collecting oils. The distribution of the sizes of drops of a residual emulsion received as a result of division process considerably changed. It is revealed that polypropylene and PTFE are the best from the point of view of efficiency of division of oil.

In work [11] model emulsions demulsified "kerosene in water" by means of thin-film microchannels. An emulsion with sizes of particles from 5 to 10 microns was received by a homogenization method. Via microchannels from PTFE with gaps from 100 to 200 microns passed repeatedly model emulsion "kerosene in water". The efficiency of a deemulgation was from $10 \%$ to $30 \%$ for one pass via the channel. At repeated passing via microchannels the efficiency of a deemulgation to $90 \%$ can be reached. In work it is defined that the efficiency of division is influenced by the stream speed, the size of particles of a disperse phase, the size of the microchannel and materials of a wall. The hydrophobic surface of the microchannel improved efficiency of division of an emulsion. The combined surface of the microchannel consisting of a polytetrafluoroethylene and stainless steel that caused various speeds of filtration is developed, and allowed droplets of an emulsion to move to a wall of PTFE and is late.

Polymeric loadings in the form of granules are used in a number of works. So, in work [13] for division of model oil emulsions 
with concentration of oil products of $790 \mathrm{mg} / \mathrm{dm} 3$ used the polymeric hydrolyzed polyacrylamide.

Division of the model emulsions supporting $\mathrm{N}$ octane in work [14] was carried out by means of the granulated polypropylene, the granulated absorbent carbon and the composite system consisting of the modified pitch (methylammonium bromide polystyrene pitch) and absorbent carbon. Results showed that the composite system has the stablest indicators of removal of oil in various service conditions because of formation of hydrogen communication between molecules of hydrocarbons and a free hydrophilic part of motionless surfactant. The efficiency of removal of oil products a composite sorbent was more than $90 \%$ of a model emulsion.

For an intensification of process of cleaning of oil-containing drains as the coalescing nozzles more than 70 types of materials are offered to application: wood fibers, ceramics, crushed stone, slag, the granulated pitches, polyurethane, the granulated styrene, peat, the made foam polystyrene, the granulated polyethylene, polypropylene, the crushed rubber and others $[15,16]$. As the coalescing materials also use various oleophylic materials, for example, cord fibers of tire covers with a diameter of $10-20$ microns [17], PTFE [10,11] and polypropylene [10].

When using of the granulated material with lack of a time (the granulated polyethylene, polytetrafluoroethylene, polyvinylchloride) placement of particles of oil is possible only between granules in a sorbent layer at the expense of capillary forces and waterrepellence. At a sufficient thickness of oil film there is an effective introduction of oil in a zone of a time, but at contact of a layer with water also absorption of water in space between granules, despite water repellency of a sorbent begins. Liquid between granules keeps due to adhesion and capillary forces therefore in settlers under the influence of gravitation there is a partial running off from a layer of the absorber of the saved-up oil (up to 93\%) $[18,19]$. The purpose of work is determination of dependence of extent of coalescent division of water oil emulsions on particle size distribution of granular PTFE.

\section{Methods}

In this work researches on determination of parameters of coalescent division of the water oil emulsions which are formed as a result of activity of petrochemical, oil-extracting and machinebuilding productions by means of granular polymers from a polytetrafluoroethylene in dynamic conditions are conducted.

For assessment of efficiency of removal of oil products from emulsions used a water oil emulsion of brand to "Inca" and a model emulsion. Dispergating of diesel fuel in $10 \%$ solution of the dodetsilsulfate of sodium and the subsequent dilution by the distilled water received a model emulsion. Coalescention was seen off on laboratory filtrational installation (fig. 1). Through the columns filled with granular polymers with the set expense passed emulsions. Filtering of emulsions through a layer of granular loading happens in two stages: delivery of particles to grains of loading and their sticking to grains. A working zone when filtering is the surface of material and space between loading grains. Height of a layer of grains of polymer - $100 \mathrm{~mm}$ in a column with an internal diameter of $10 \mathrm{~mm}, 150 \mathrm{~mm}$ long, the mass of the coalescing filler was 2 grams. The size of grains of polymer was from 0,5 to $3 \mathrm{~mm}$. Concentration of oil products in a water oil emulsion of brand to "Inca" made $828 \mathrm{mg} / \mathrm{dm} 3$, and a model emulsion from diesel fuel - $635 \mathrm{mg} / \mathrm{dm} 3$.

Regeneration of the filtering loading was carried out by washing with four-chloride carbon.

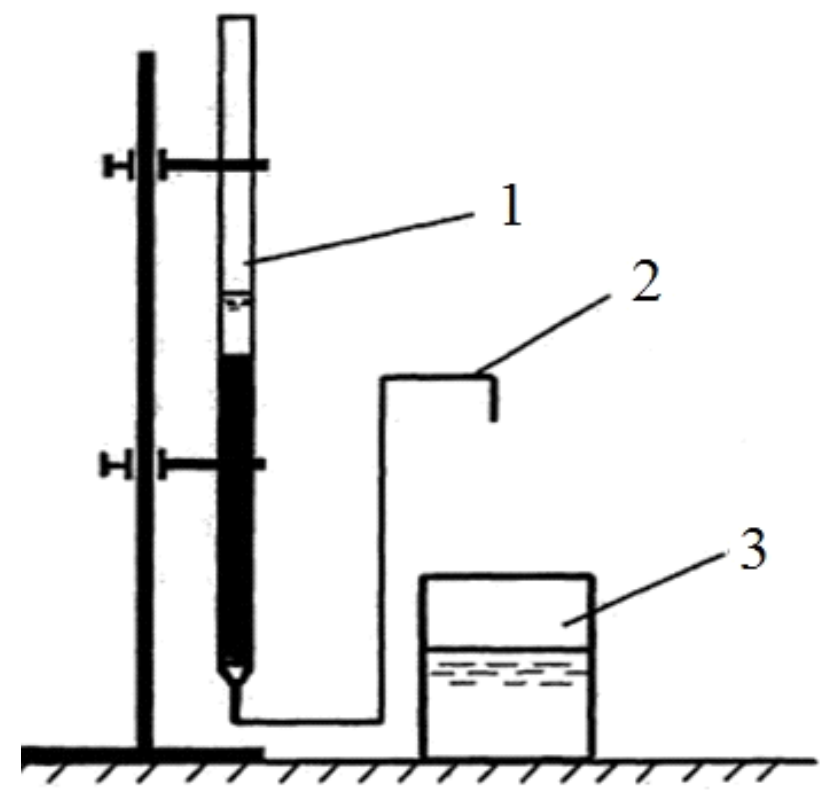

Figure 1. Scheme of laboratory filtrational installation: 1 - a column with adsorbent; 2 - withdrawal of a filtrate; 3 - glass

The size of particles of a disperse phase of emulsions, determined by method of dynamic light scattering (DLS), and $\zeta$-potential - - - - . - - - a light scattering method with the analysis of phases (PALS) by the analyzer of NanoBrook Omni brand [20].

As key indicators of coalescent division of an emulsion productivity and extent of division of water oil emulsion which was calculated as the relation of content of the oil products in an emulsion before division defined by a concentration meter of the KN-3 brand was considered?

The efficiency of cleaning of installation was determined by extent of removal oil products and the weighed substances on a formula:

$$
\varphi=\left(\mathrm{C}_{\mathrm{f}}-\mathrm{C}_{\mathrm{p}}\right) / \mathrm{C}_{\mathrm{f}},
$$

where $\mathrm{Cf}$ and $\mathrm{Sp}$ - concentration of pollutant in the initial and purified solution, respectively.

\section{Results and discussion}

In the figure 2 and results of measurements of the size of particles and $\zeta$-potential of a disperse phase of the studied emulsions are presented in table 1.

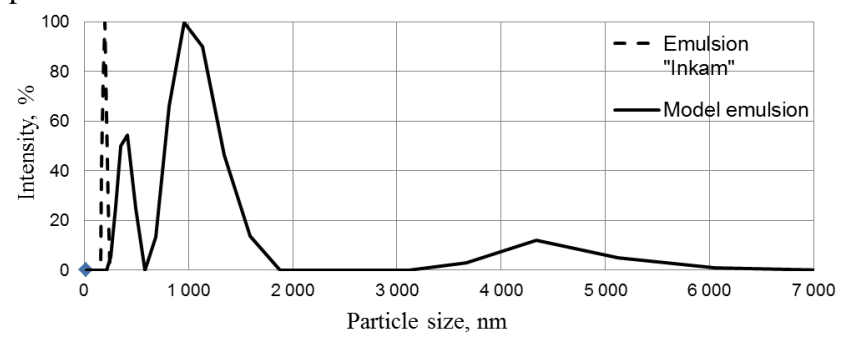

Figure 2. Schedules of distribution of the size of particles of a disperse phase of emulsions: (continuous line) model emulsion and (dashed line) water oil emulsion to "Inca".

On the basis of the data submitted in the figure 3 the emulsion to Inca is polydisperse system with sizes of particles of $155-230$ nanometers. $\zeta$-potential at all studied emulsions negative. As a rule, with increase in the size of particles, $\zeta$-potential between particles decreases; the $\zeta$-potential of particles is less, the stronger 
particles stick together, integrated with each other, there is a loss of a deposit. Model emulsion less stable and size of particles of a disperse phase from 252 to 6055 nanometers. In the figure 3 the dependence of speed of filtration through a column from the size of grains of polymer is presented.

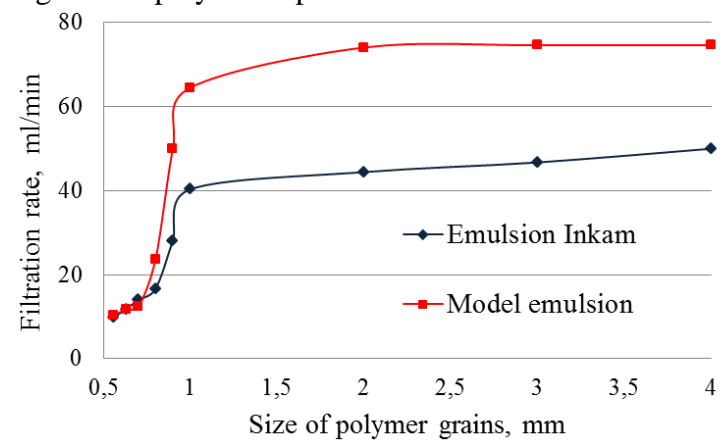

Figure 3. Schedules of dependence of speed of filtration of an emulsion on the size of grains of a polytetrafluoroethylene.

Speed of filtration of an emulsion through a column filled with polymer grains minimum at the size of grains less than $0,5 \mathrm{~mm}$. At increase in the size of grains of polymer up to $1 \mathrm{~mm}$ filtration speed sharply increases by 4-6 times. Speed filtration of an emulsion through polymer grains in the range of sizes from 1 to $4 \mathrm{~mm}$ fall off slightly. At filtration of a model emulsion with sizes of particles of oil of 252-6000 nanometers the speed of filtration is $20 \mathrm{ml} / \mathrm{min}$. higher than at filtration of an emulsion to Inca with a size of particles of 155-230 nanometers. Probably large particles of oil are late at the beginning of a column more intensively and water freely passes through a column. Extent of cleaning of oil products, is confirmed by results of the chemical analysis of tests of the initial and purified liquid. Results of cleaning of emulsions with method of a coalescention on grains of a polytetrafluoroethylene are presented in the figure 4.

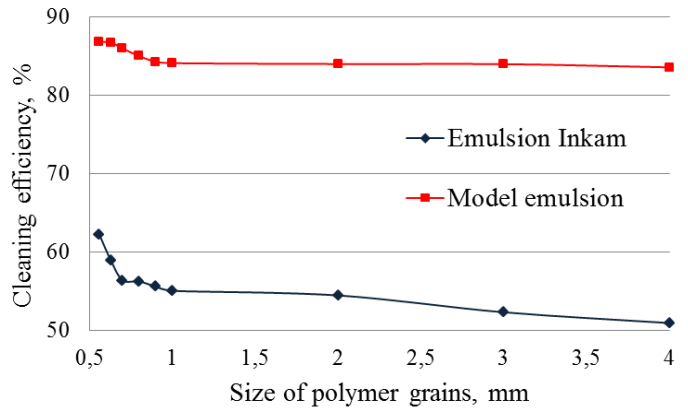

Figure 4. Schedules of dependence of extent of cleaning (on an indicator oil products) emulsions from the size of grains of a polytetrafluoroethylene.

The efficiency of cleaning of a model emulsion of oil products was $83-86 \%$ depending on the sizes of grains of polymer, and extent of removal of oil products to "Inca" not high also makes only $48-68 \%$ of an emulsion. Particles of a disperse phase of an emulsion to "Inca" have the smaller size, namely $155-230$ nanometers that complicate aggregation of particles on the surface of grains of polymer. Increase in the size of grains of polymer leads to decrease in efficiency of cleaning of oil products.

By results of a research the emulsion to "Inca" is polydisperse system with sizes of particles of $155-230$ nanometers, $\zeta$-potential at an emulsion negative- $71 \mathrm{mV}$. The size of particles of a disperse phase of the model emulsion received by dispergating of diesel fuel in 30\% solution of the dodetsilsulfat of sodium was 252-6055 nanometers. Speed of filtration of these emulsions through a column depends on the size of grains of a polytetrafluoroethylene, with increase in the size of particles the speed of filtration increases, but the efficiency of removal of oil products decreases. The disperse system of a model emulsion is less stable, than the brand emulsion to "Inca" that leads to adhesion of particles on the surface of polymer because of water-repellence, leads further to ag- gregation of particles therefore also efficiency of cleaning high. According to literary data placement of particles of oil products happens between granules in a sorbent layer at the expense of capillary forces and water-repellence $[15,16]$. The size of grains of polymer in the range from 0,5 to $1 \mathrm{~mm}$ is optimum at which high extent of removal of particles of oil products, with the minimum speed of filtration is observed.

Table 1. Values of the size of particles and $\zeta$-potential of a disperse phase of emulsions

\begin{tabular}{|c|c|c|}
\hline Emulsion & Size of particles, nanometer & $\begin{array}{c}\zeta \text {-potential, } \\
\mathrm{mV}\end{array}$ \\
\hline Emulsion to "Inca-1" & $155-230$ & $-73 \pm 7$ \\
\hline Model emulsion & $252-493 ; 689-1580 ; 3667-6055$ & $-31 \pm 3$ \\
\hline
\end{tabular}

\section{Conclusions}

The efficiency of removal of particles of oil products from an emulsion depends on the size of grains of a polytetrafluoroethylene, the size of grains is less, and the efficiency is higher. Also the efficiency of removal of oil products depends on the size of particles of a disperse phase of emulsions, and on value of $\zeta$ potential of an emulsion as pushing away measures between particles of a disperse phase, that is an indicator of stability of system. The process of a coalescention proceeding on the surface of grains of hydrophobic polymer during filtration through a column in dynamic conditions is recommended to use for preliminary cleaning of oil products of sewage, unstable oil or oil emulsions with sizes of a disperse phase more $0,5 \mathrm{mkm}$

\section{Acknowledgement}

This work was supported by scientific the research grant of Kazan Federal University.

\section{References}

[1] Fazullin D.D., Mavrin, Sokolov M.P. Utilization of waste lubricating-cooling fluids by membrane methods/Chemistry and Technology of fuels and Oils. 2015. Vol. 51. No 1. River 93-98.

[2] Fazullin D.D., Mavrin, G. V., Shaikhiev I.G. Modified PTFE-PANI Membranes for the Recovery of Oil Products from Aqueous Oil Emulsions/Petroleum Chemistry. 2017. Vol. 57. No 2. River 165 171.

[3] Fazullin, D. D., Mavrin, G. V., Nasyrov, I. A. Dynamic membranes of nylon-PTFE for separation of water-oil emulsions. Journal of Fundamental and Applied Sciences. 2017. Volume. 9 Issue. 1S. Pages: 1441-1449.

[4] Sunil Lalchand Kokal. Crude Oil Emulsions: A State-Of-The-Art Review/Society of Petroleum Engineers. 2005. Vol. 20. No 1. River 93-98.

[5] Isaacs E.E., Huang H., Babchin A.J., Chow R.S. Electroacoustic method for monitoring the coalescence of water-in-oil emulsions/Colloids and Surfaces. 1990. Vol. 46. No. 2. P. 177-192.

[6] Tetsuji Hirato, Kazuya Koyama, Takahiro Tanaka, Yasuhiro Awakura. Hiroshi Majima Demulsification of Water-in-Oil Emulsion by an Electrostatic Coalescence Method/Materials Transactions. 1991. Vol. 32. No. 3. P. 257-263.

[7] Soumitra Maiti, Mishra I.M., Bhattacharya S.D., Joshi J.K. Removal of oil from oil-in-water emulsion using a packed bed of commercial resin/Colloids and Surfaces A: Physicochemical and Engineering Aspects. 2011. Vol. 389. No. 1-3. P. 291-298.

[8] Stalemate. 124672 Russian Federation, MPK C02F1/00 Sewage treatment unit and technological liquids / Fazullin D.D., Mavrin, Nasyrov I.A. - 2012118948/05; zayavl. 05.05.2012; opubl. 10.02.2013.

[9] Paixao M.V.G., Balaban R.D., Application of guar gum in brine clarification and oily water treatment/International journal of biological macromolecules. 2018. Vol. 108. River 119-126

[10] Wang Shuo, Qin Wei, Dai Youyuan. Separation of Oil Phase from Dilute Oil/Water Emulsion in Confined Space Apparatus. Chinese journal of chemical engineering. 2012. Vol. 20. Issue. 2. River 239245 . 
[11] Xiao Chen, Houfang Lu, Wei Jiang. De-emulsification of Kerosene/Water Emulsions with Plate-Type Microchannels. Industrial \& engineering chemistry research. 2010. Vol. 49. Issue. 19. P. 9279 9288

[12] Fazullin D. D., Mavrin G. V. Coalescence of water-oil emulsions on thin-layered PVC plates/Turkish online journal of design art and communication. 2017 Vol. 7. P. 1686-1692.

[13] 1 Shatilova L.M., Borisova V.V., Kasatkina O.A. (2018). Representation of the linguistic and cultural concept "lie" in the French and Russian language picture of the world, 34(85), Pp. 194-212.

[14] 2 Bakhyt S., Kalimbetov B., Khabibullayev Z. (2018). Possibilities of Mathematical Problems in Logical Thinking, Development of Secondary Education Pupils, 34(85), P.p. 321-338..

[15] Jingquan Li, Yongan Gu. Coalescence of oil-in-water emulsions in fibrous and granular beds/Separation and Purification Technology. 2005. Vol. 42. No 1. P. 1-13.

[16] Swarna Bansal and others. Effect of fibrous filter properties on the oil-in-water-emulsion separation and filtration performance/Journal of Hazardous Materials. 2011. Vol. 190. No 1-3. P. 45-50.

[17] Adelshin A. B. Intensification of processes of hydrodynamic purification of oil-containing sewage: Tech. Sci. PhD: 05.23.04. / Adelshin Azat Bilyalovich. SPb., 1998. 74 pages.

[18] Vu Van TUAN Communicative Competence of the Fourth Year College Students: Basis for proposed English Language Program, Astra Salvensis, Supplement No. 2, 2017,p. 45

[19] [1] Irina MALGANOVA, Andrey ERMAKOV, Development of heating Devices from Polypropylene, Astra Salvensis, Supplement No. 2, 2017, p. 93 